\title{
Relación entre desempeño docente y satisfacción en enfermeras egresadas de maestría en una universidad pública
}

Relationship between teaching performance and the satisfaction level of the graduates from master in nursing in one public university

\author{
Lucio Similiano Reyes Pariona 1,2,a
}

\section{RESUMEN}

Objetivo: Determinar la relación entre el desempeño de los docentes de maestría en enfermería con el nivel de satisfacción de enfermeras egresadas en una universidad Pública de Lima. Material y Métodos: Estudio cuantitativo, correlacional y transversal. La población estuvo constituida por 30 egresados de maestría en enfermería de una universidad pública de Lima- Perú. Se validaron 2 cuestionarios; para desempeño docente y nivel de satisfacción, obteniendo alfa de Crombach de 0,83 y 0,89 respectivamente. Resultados: Los participantes se caracterizaron por tener entre 31 y 35 años $(40 \%)$, de sexo femenino $(83,3 \%)$ y ser solteros $(70 \%)$. Respecto a desempeño docente predominó el nivel regular $(60 \%)$ y nivel medio de satisfacción $(53,3 \%)$. Se encontró correlación positiva media de 0,62 entre las variables ( $\mathrm{p}<0,001)$. Conclusiones: Parece ser que el desempeño de los docentes de maestría en enfermería se relaciona significativamente con el nivel de satisfacción de los egresados, por lo que es sugerente tomar en cuenta los resultados con el fin de elevar la calidad educativa en este nivel de estudio.

PALABRAS CLAVE: Desempeño del rol, docentes de enfermería, satisfacción personal, estudiante de enfermería.

\section{SUMMARY}

Objective: To determine the relationship between the professors performance and the satisfaction level of Graduates from Master in Nursing in one public university in Lima. Material and methods: Quantitative, cross-sectional and descriptive-correlational study. The population consisted of 30 Graduates from Master in Nursing in one public university in Lima, Peru. Two questionnaires were validated; one for professor's performance and one for satisfaction level, obtaining a Cronbach's Alpha of 0.83 and 0.89 respectively. Results: The participants were characterized by being $31-35$ years old $(40 \%)$, female $(83.3 \%)$ and being single $(70 \%)$. Concerning the professors performance the regular level $(60 \%)$ and average level of satisfaction (53.3\%) predominated. An average positive correlation of 0.62 between the two variables $(\mathrm{p}<0.001)$ was found. Conclusions: The performance of Professors from Master in Nursing is significantly related to the satisfaction level of the Graduates, so it is important to take into account the results in order to raise the education quality at this level of study.

KEY WORDS: Role playing; faculty; nursing; personal satisfaction; students; nursing.

Hospital Nacional Hipólito Unanue. Lima, Perú.

Facultad de Medicina, Universidad Nacional Mayor de San Marcos. Lima, Perú.

Licenciado en enfermería 


\section{INTRODUCCIÓN}

La educación superior a nivel universitario sea de índole pública o privada, juega un papel de vital importancia en la sociedad ya que forma seres humanos que contribuyen a transformarla, por ello el desempeño del docente en este nivel es un factor fundamental de la calidad educativa, ya que tienen la responsabilidad de guiar y formar ciudadanos competentes capaces de responder eficientemente a la problemática actual y de futuro del mundo globalizado.

Por su parte, el estudiante enfermero se ha convertido en la piedra angular del proceso enseñanzaaprendizaje, ya que es el actor principal de generar mayores posibilidades de desarrollo en un país y de la calidad de vida de la persona, familia y comunidad. En ese contexto su satisfacción se convierte en un indicador relacionado con el cumplimiento de metas, aprendizajes, expectativas y calidad del servicio educativo recibido (1).

En relación con ello, diversos organismos internacionales agregan que la educación universitaria es un factor fundamental para el desarrollo humano sostenible y racional, por lo que plantean que deben responder a las necesidades de la sociedad, innovando en sus funciones y organización, esto quiere decir que la enseñanza a este nivel debe garantizar la calidad educativa de los estudiantes, para que ellos puedan responder oportunamente a cualquier situación problemática (2).

Por su parte, el Ministerio de Educación ha establecido la política de Aseguraramiento de la Calidad Educativa, señalando que la universidad es una comunidad académica orientada a la investigación y a la docencia, que brinda formación humanista, científica y tecnológica con una clara conciencia de nuestro país como realidad multicultural.

En este marco, la nueva Ley Universitaria 30220, promueve la calidad educativa de las instituciones universitarias como entes fundamentales del desarrollo nacional, de la investigación y de la cultura; estableciendo fines, funciones y principios orientados a la libertad de cátedra; el espíritu crítico; la pertinencia; el compromiso con el crecimiento del país y el mejoramiento constante de la calidad académica, entre otros (3).

En este sentido, a nivel internacional existen estudios que evalúan la satisfacción del estudiante en relación con el desempeño docente y calidad educativa realizados con estudiantes de pregrado en profesiones como administración (4), educación (5), y enfermería (6). En el ámbito nacional hay diversos estudios realizados en estudiantes de educación básica regular (7), estudiantes de enfermería de pregrado $(8,9)$, de posgrado, pero en educación $(10,11)$; por lo que se evidencia en la búsqueda que hay un vacío de conocimiento en esta temática.

El presente estudio tuvo como objetivo aportar al conocimiento en el campo de enfermería a nivel de postgrado sobre desempeño docente y satisfacción en enfermeras egresadas de maestría en una universidad pública, para que los resultados puedan ser considerados por las autoridades a fin de que tomen decisiones y establezcan estrategias que permitan fortalecer los aspectos positivos y/o subsanar debilidades en mejora de la calidad educativa en ese nivel de estudio, y puedan contar con un material de referencia para futuros estudios en otras líneas de posgrado, en marco del aseguramiento de la calidad educativa.

\section{MATERIAL Y MÉTODOS}

Estudio de enfoque cuantitativo, descriptivo, correlacional y transversal. La población de estudio estuvo conformada por 30 enfermeras egresadas de maestría con mención en docencia y gestión de una universidad pública de Lima, por el reducido número de sujetos, no se realizó ninguna técnica de muestreo. Los criterios de inclusión fueron enfermeras egresadas del programa de maestría, que aceptaran participar en el estudio de forma voluntaria firmando el consentimiento informado.

Para recolectar los datos de desempeño docente y satisfacción, se validaron 2 escalas tipo Likert modificadas, las cuales fueron fundamentadas en el marco teórico referencial de autores como Gento (12) y Arregui (13) respectivamente. El instrumento modificado para medir el desempeño docente consta de 22 reactivos distribuidos en 3 dimensiones. Cada ítem tuvo 4 alternativas de respuesta: nunca, a veces, casi siempre, siempre. Para el nivel de satisfacción, la escala modificada estuvo constituida por 20 reactivos en 3 dimensiones establecidas y cada ítem cuenta con 4 alternativas de respuesta: muy insatisfecho, poco satisfecho, bastante satisfecho, muy satisfecho. Para el proceso de validación se realizó prueba de jueces analizada con prueba binomial y para evaluar la confiabilidad se realizó la prueba piloto a un grupo de 
10 enfermeras egresadas de la maestría, se analizaron los datos mediante alfa de Crombach, obteniéndose 0,83 para el desempeño docente y 0,89 en el nivel de satisfacción. La aplicación de los dos instrumentos duró 30 minutos en promedio.

Se utilizó el paquete estadístico SPSS versión 23, para luego ser analizados y clasificados en tres niveles para cada variable, a través de la campana de Gauss, donde se halló la sumatoria total de los puntajes, con la cual se obtuvo la media, la desviación estándar, valor mínimo y máximo con una constante de 0.75 , ello permitió obtener los límites de la campana y ser agrupadas, para el desempeño docente en bueno (7588 ), regular (59-74) y deficiente (46-58) y para el nivel de satisfacción en nivel alto (66-75), nivel medio (51$65)$ y nivel bajo (40-50). Asimismo, para determinar la relación entre ambas variables de estudio, se utilizó la prueba paramétrica coeficiente de Correlación de Pearson, luego de contrastar la normalidad de los datos mediante la prueba de Shapiro-Wilk.

Se obtuvieron los permisos institucionales de la universidad pública, así como la respectiva autorización por su comité de ética. A los participantes se solicitó consentimiento informado para salvaguardar los principios bioéticos.

\section{RESULTADOS}

En la tabla 1 se evidencia que la mayoría de participantes son de sexo femenino $(83,3 \%)$, solteros (70\%) y tienen entre 31 a 35 años (40\%).

En la tabla 2 se aprecia la variable desempeño docente a detalle con los resultados encontrados en sus 3 dimensiones. Para capacidades pedagógicas, se obtuvo que los docentes prioritariamente presentan desempeño regular, debido a que promueven el desarrollo de habilidades como análisis, síntesis y creatividad (70\%), demuestran dominio de los temas que imparten $(66,7 \%)$ y habilidades pedagógicas en las clases $(63 \%)$. En cuanto a la disposición para la labor docente, se encontró predominio regular, ya que los docentes relacionan la teoría con la práctica para el aprendizaje significativo de los estudiantes de maestría (73\%), aplican las tecnologías educativas en sus clases $(70 \%)$ e incentivan el aprendizaje de los maestrandos $(66,7 \%)$. En responsabilidad laboral si bien fue de regular desempeño, destacó que los docentes denotan saberes profesionales para el nivel de postgrado $(66,7 \%)$.
En cuanto a la satisfacción en enfermeras egresadas de maestría, la tabla 3 muestra los resultados en sus 3 dimensiones, siendo predominante el nivel medio. Para el proceso de enseñanza-aprendizaje; la comunicación transversal docente-estudiante $(70 \%)$, el dominio del contenido de las asignaturas $(66,7 \%)$ y tono de voz claro e armónico en el aula (60\%). En la planificación del proceso de enseñanza-aprendizaje; la estrategia de evaluación objetiva de los aprendizajes $(76,7 \%)$, la organización de los planes en clases $(73,3 \%)$, uso de diversas técnicas de enseñanza-aprendizaje $(60,3 \%)$ y efectividad de las tics en el aprendizaje significativo $(50 \%)$. En el apoyo y trato docente; destacaron el trato cálido humano $(76,7 \%)$, el respeto por las opiniones de los maestrandos $(73,3 \%)$ y el apoyo moral $(66,7 \%)$. En el gráfico 1 se observa predominio regular del desempeño docente $(60 \%)$ y nivel medio de satisfacción en enfermeras egresadas $(53,3 \%)$. Como el estudio pretendió determinar la relación entre ambas variables de estudio mencionadas, se encontró correlación media positiva $(\mathrm{r}=0,62$ y valor $\mathrm{p}<0,001)$.

Tabla 1. Características de enfermeras egresadas de maestría de una universidad pública

\begin{tabular}{llcc}
\hline \multicolumn{2}{l}{ Características } & n & \% \\
\hline Edad: & De 25 - 30 años & 10 & 33,3 \\
& De31 - 35 años & 12 & 40,0 \\
& $>$ de 35 años & 8 & 26,7 \\
Sexo: & Femenino & 25 & 83,3 \\
& Masculino & 5 & 16,7 \\
E. Civil: & Soltero & 21 & 70,0 \\
& Conviviente & 5 & 16,7 \\
& Casado & 4 & 13,3 \\
\hline
\end{tabular}

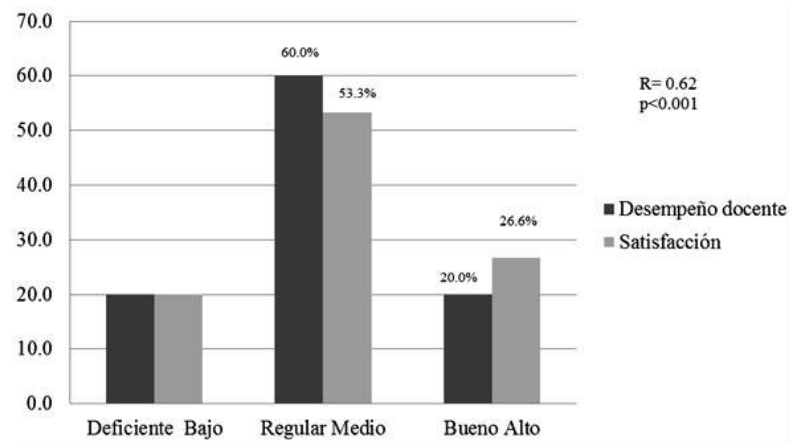

Gráfico 1. Correlación entre el desempeño docente y nivel de satisfacción de enfermeras egresadas de la maestría en una universidad pública de Lima. 
Tabla 2 . Desempeño de los docentes de maestría según dimensiones de una universidad pública

ESCALA

ITEMS

Bueno Regular Deficiente

\section{Capacidades pedagógicas}

1. Los docentes demuestran dominio de los temas que imparten.

$\begin{array}{llllll}\text { n } & \% & \text { n } & \% & \text { n } & \%\end{array}$

2. Los docentes exponen temas actualizados.

3. Los docentes retroalimentan los contenidos impartidos en las clases.

4. Los docentes demuestran habilidades pedagógicas en las clases.

5. Los docentes planifican las clases haciendo uso de las diferentes estrategias didácticas innovadoras.

6. Los docentes al inicio de cada clase comunican claramente los objetivos de aprendizaje.

7. Los docentes promueven el desarrollo de habilidades en los maestrandos (análisis, síntesis y creatividad).

\begin{tabular}{|c|c|c|c|c|c|}
\hline 6 & 20 & 20 & 66,7 & 4 & 13,3 \\
\hline 8 & 26,7 & 151 & 50 & 7 & 23,3 \\
\hline 5 & 16,7 & 6 & 53,3 & 9 & 30 \\
\hline 6 & 20 & 19 & 63 & 5 & 16,7 \\
\hline 7 & 23 & 16 & 53,3 & 7 & 23 \\
\hline 5 & 16,7 & 16 & 53,3 & 9 & 30 \\
\hline 5 & 16,7 & 21 & 70 & 4 & 13,3 \\
\hline 8 & 26,7 & 16 & 53,3 & 6 & 20 \\
\hline $\mathrm{n}$ & $\%$ & $\mathrm{n}$ & $\%$ & $\mathrm{n}$ & $\%$ \\
\hline 9 & 30 & 15 & 50 & 6 & 20 \\
\hline 6 & 20 & 20 & 66,7 & 4 & 13,3 \\
\hline 5 & 16,7 & 21 & 70 & 4 & 13,3 \\
\hline 4 & 13,3 & 22 & 73,3 & 4 & 13,3 \\
\hline 4 & 13,3 & 21 & 70 & 5 & 16,7 \\
\hline 7 & 23,3 & 21 & 70 & 2 & 6,7 \\
\hline 6 & 20 & 20 & 66,7 & 4 & 13,3 \\
\hline 2 & 6,7 & 17 & 56,7 & 11 & 36,6 \\
\hline $\mathrm{n}$ & $\%$ & $\mathrm{n}$ & $\%$ & $\mathrm{n}$ & $\%$ \\
\hline 6 & 20 & 16 & 53,3 & 8 & 26,7 \\
\hline 9 & 30 & 18 & 60 & 3 & 10 \\
\hline 9 & 30 & 15 & 50 & 6 & 20 \\
\hline 4 & 13,3 & 17 & 56,7 & 9 & 30 \\
\hline 8 & 26,7 & 18 & 60 & 4 & 13,3 \\
\hline 8 & 26,7 & 20 & 66,7 & 2 & 6,7 \\
\hline
\end{tabular}

8. Los docentes mantienen una comunicación horizontal en el aula con los maestrandos.

\section{Disposición para la labor docente}

9. Los docentes muestran trato equitativo con todos los maestrandos.

10. Los docentes incentivan el aprendizaje de los maestrandos.

11. Los docentes valoran los esfuerzos y logros de los maestrandos.

12. Los docentes relacionan la teoría con la práctica para el aprendizaje significativo.

13. Los docentes aplican las tecnologías educativas en sus clases.

14. Los docentes denotan autonomía en las clases impartidas.

15. Los docentes motivan a los maestrandos en su aprendizaje permanentemente.

16.Los docentes brindan apoyo emocional a los maestrandos cuando lo necesitan.

\section{Responsabilidad laboral}

17.Los docentes brindan apoyo emocional a los maestrandos cuando lo necesitan.

18.Los docentes muestran interés por el aprendizaje de los maestrandos.

19.Los docentes cumplen con todos los contenidos del silabo.

20.Los docentes comparten referencias bibliográficas actualizadas con los maestrandos.

21.Los docentes denotan profesionalismo en cada clase que imparten.

22.Los docentes denotan saberes profesionales para el nivel de postgrado. 
Tabla 3. Nivel de satisfacción de enfermeras egresadas de maestría según dimensiones de una universidad pública

\begin{tabular}{|c|c|c|c|c|c|c|}
\hline \multirow{3}{*}{ ITEMS } & \multicolumn{6}{|c|}{ ESCALA } \\
\hline & \multicolumn{2}{|c|}{ Nivel alto } & \multicolumn{2}{|c|}{ Nivel medio } & \multicolumn{2}{|c|}{ Nivel bajo } \\
\hline & $\mathbf{n}$ & $\%$ & $\mathbf{n}$ & $\%$ & $\mathbf{n}$ & $\%$ \\
\hline \multicolumn{7}{|l|}{ Satisfacción con el proceso de enseñanza - aprendizaje } \\
\hline 1.Dominio del contenido de las asignaturas. & 5 & 16,7 & 20 & 66,7 & 5 & 16,7 \\
\hline 2.Actualización, preparación de los docentes. & 4 & 13,3 & 19 & 60,3 & 7 & 23,3 \\
\hline 3.Objetividad de la evaluación del aprendizaje. & 4 & 13,3 & 20 & 66,7 & 6 & 20 \\
\hline 4.Logro de objetivos del aprendizaje. & 4 & 13,3 & 16 & 53,3 & 10 & 33,3 \\
\hline 5.Estrategias de aprendizaje utilizadas. & 5 & 16,7 & 14 & 46,7 & 11 & 36,7 \\
\hline 6.Materiales didácticos disponibles. & 4 & 13,3 & 17 & 56,7 & 9 & 30 \\
\hline 7.Comunicación transversal docente-maestrando. & 5 & 16,7 & 21 & 70 & 4 & 13,3 \\
\hline 8.Tono de voz claro e armónico en el aula. & 8 & 26,8 & 18 & 60 & 4 & 13,3 \\
\hline Satisfacción con la planificación del Proceso de E-A & $\mathrm{n}$ & $\%$ & $\mathrm{n}$ & $\%$ & $\mathrm{n}$ & $\%$ \\
\hline 9.Planificación de evaluación objetiva de los aprendizajes. & 2 & 6,7 & 23 & 76,7 & 5 & 16,7 \\
\hline 10.Organización de los planes de clases. & 3 & 10 & 22 & 73,3 & 5 & 16,7 \\
\hline 11.Uso de diversas técnicas de enseñanza-aprendizaje. & 1 & 3,3 & 19 & 60,3 & 10 & 33,3 \\
\hline 12.Expectativas de aprendizaje logrados. & 2 & 6,7 & 19 & 60,3 & 9 & 30 \\
\hline 13.Tecnología educativa de enseñanza -aprendizaje. & 3 & 10 & 19 & 60,3 & 8 & 26,8 \\
\hline 14.Efectividad de las tics en aprendizaje significativo. & 2 & 6,7 & 15 & 50 & 13 & 43,3 \\
\hline Satisfacción con el apoyo y trato docente & $\mathrm{n}$ & $\%$ & $\mathrm{n}$ & $\%$ & $\mathrm{n}$ & $\%$ \\
\hline 15.Disposición de escucha activa. & 4 & 13,3 & 19 & 60,3 & 7 & 23,3 \\
\hline 16.Disposición de clarificar dudas en clase. & 6 & 20 & 19 & 60,3 & 5 & 16,7 \\
\hline 17.Respeto por las opiniones de los maestrandos. & 4 & 13,3 & 22 & 73,3 & 4 & 13,3 \\
\hline 18.Trato cálido humano de los docentes. & 4 & 13,3 & 23 & 76,7 & 3 & 10 \\
\hline 19.Apoyo moral a los maestrandos. & 3 & 10 & 20 & 66,7 & 7 & 23,3 \\
\hline 20.Apoyo académico que brindan los docentes fuera del aula. & 2 & 6,7 & 20 & 66,7 & 8 & 26,8 \\
\hline
\end{tabular}

\section{DISCUSIÓN}

A partir de los hallazgos obtenidos se evidencia correlación positiva media por lo que parece ser que el desempeño de los docentes de Maestría en enfermería se relaciona significativamente con el nivel de satisfacción de las enfermeras egresadas. Ello guarda relación con otros estudios donde también encontraron relación estadísticamente significativa como de Falcón, que evaluó el desempeño docente $\mathrm{y}$ satisfacción estudiantil (7) $\mathrm{y}$ en el caso de Tolentino, quien investigó el desempeño didáctico y académico del docente con el grado de satisfacción de los estudiantes (10). Cabe destacar que el estudio de Blancas, determinó correlación moderada entre el proceso de enseñanza-aprendizaje y el nivel de satisfacción de los estudiantes (9); aunque este estudio fue evaluado con una metodología diferente.

Asimismo, se identificó entre los sujetos encuestados, que el desempeño de los docentes de maestría en enfermería es regular, lo cual significa que no estarían desempeñándose según las exigencias requeridas para cumplir con los estándares de calidad necesarios en una maestría; por lo que se requeriría establecer una serie de estrategias tendientes a mejora en su quehacer en el aula, que garanticen la calidad educativa, más de quienes guiarán a los estudiantes en el proceso de aprendizaje, el cuidado de la persona, familia y comunidad en bien de una sociedad sostenible y armónica, este último guarda relación con lo que señala Rivas, quien encontró que 
el desempeño docente de enfermería en el pregrado de una universidad pública es regular (14). Al respecto Montenegro refiere que "el desempeño docente es el cumplimiento de sus funciones, y se halla determinado por factores asociados al propio docente, al estudiante y al entorno". Así, el desempeño se ejerce en los diferentes espacios del contexto educativo, teniendo como fin garantizar la calidad educativa de los estudiantes en todo nivel de estudio, considerando nuestra realidad multicultural (15).

En cuanto al nivel de satisfacción de las enfermeras egresadas de la maestría, se identificó que la mayoría de los sujetos encuestados evidencia nivel medio de satisfacción, visibilizando que el desempeño de los docentes no estaría satisfaciendo en su totalidad, las necesidades y cumpliendo a sus expectativas de aprendizaje en enfermeras, poniendo en riesgo la calidad educativa. Ello discrepa con Lepiani y Dueñas quienes mencionan que los estudiantes se encuentran satisfechos con los diferentes aspectos del proceso formativo adaptado al espacio europeo (6), posiblemente debido a que se trata de un estudio en diferente realidad del contexto educativo y cultual. Al respecto Jiménez refiere que la satisfacción del estudiante constituye uno de los indicadores más importantes para medir la calidad de la enseñanza y un elemento clave en la valoración de la calidad de la educación, ya que refleja la eficiencia de los servicios académicos y administrativos, su satisfacción con las unidades de aprendizaje, con las interacciones con su profesor y compañeros de clase, así como con las instalaciones y equipamiento del contexto educativo (5).

Cabe destacar que, si bien se obtuvo desempeño regular en el docente, las egresadas destacaron que los docentes son puntuales, muestran interés por el aprendizaje del estudiante, comparten referencias bibliográficas actualizadas que denotan profesionalismo en cada clase que imparten.

Estos hallazgos son coherentes con lo que Tejedor y García mencionan, el desempeño docente significa cumplir con responsabilidad, hacer lo mejor posible aquello que se tiene encomendado, es el cumplimiento del trabajo de manera interrelacionada e intencionada los saberes, las habilidades, las actitudes y valores de que dispone el educador para el ejercicio de su función (16). Y con Reymer, quien agrega que en el terreno educativo "la satisfacción se concibe como aquella respuesta del estudiante frente a un conjunto de servicios que tienen como fin último producir un aprendizaje real, significativo y contextualizado" (11). En cuanto a las limitaciones cabe mencionar que, la población objeto de estudio no permitió una amplia generalización de los resultados debido a su tamaño, por lo que los hallazgos del estudio están limitados solo a las enfermeras egresadas de dicha institución. Así mismo reconocemos que se podría establecer estudios posteriores con metodología multivariante para mejorar el control de otras variables no identificadas en el estudio. A pesar de ello, los resultados siguen siendo válidos desde una perspectiva de investigación exploratoria.

\section{CONCLUSIONES}

El desempeño de los docentes de maestría en enfermería en una universidad pública de Lima es predominantemente regular y nivel medio de satisfacción; Así mismo que las variables desempeño docente y nivel de satisfacción se relacionan significativamente.

Correspondencia:

Lucio Similiano Reyes Pariona

Correo electrónico: reyesparionalucio@gmail.com

\section{REFERENCIAS BIBLIOGRÁFICAS}

1. Tejedor FJ. Evaluación Del Desempeño Docente. Revista Iberoamericana de Evaluación Educativa. 2012; 5 (1): 319-327. (Fecha de acceso 15 de agosto del 2017) Disponible en: http://www.rinace.net/riee/ numeros/vol5-num1_e/art24.pdf

2. Vicerrectorado Académico, Universidad Nacional Mayor de San Marcos. Modelo Educativo San Marcos. Lima: Universidad Nacional Mayor de San Marcos; 2013. (Fecha de acceso 15 de agosto del 2017) Disponible en: http://viceacademico.unmsm. edu.pe/archivos/MODELO \%20EDUCATIVO\%20 2013 para_V icerrectorado.pdf

3. Congreso de la República del Perú. Ley Universitaria №30220 del Perú. Lima: Diario Oficial El Peruano; 9 de julio del 2014. (Fecha de acceso 15 de noviembre del 2017) Disponible en: https://www.sunedu.gob.pe/ wp-content/uploads/2017/04/Ley-universitaria30220.pdf

4. Surdez EG, Sandoval MC, Lamoyi LC. Satisfacción estudiantil en la valoración de la calidad educativa universitaria. Educación y Educadores Educación y Educadores; 21(1): 9-26.

5. Jiménez A, Terriquez B, Robles F. Evaluación de la satisfacción académica de los estudiantes de la Universidad Autónoma de Nayarit. Rev Fuente. 2011; 6(6):46-56. 
6. Lepiani I, Dueñas M, Medialdea M, Bocchino A. Satisfacción de estudiantes de enfermería con el proceso formativo adaptado al Espacio Europeo de Educación Superior. Enfermería Docente. 2013; 101: 22-8. (Fecha de acceso 15 de noviembre del 2017) Disponible en: http://www.juntadeandalucia. es/servicioandaluzdesalud/huvvsites/default/files/ revistas/ED-101-06.pdf

7. Falcón G. Desempeño docente y satisfacción académica en estudiantes de educación secundaria. Con Ciencia EPG. 2016; 1(1): 42-8. DOI 10.32654/ CONCIENCIAEPG.1-1.3

8. Urure I, Campos R, Ventura CT, Curasi O, Gómez L. Satisfacción de los estudiantes con la calidad de enseñanza de los docentes de la Facultad de Enfermería de la Universidad Nacional San Luis Gonzaga de Ica, octubre 2013 - octubre 2014. (Fecha de acceso 15 de noviembre del 2017) Disponible en: http://www.unica.edu.pe/alavanguardia/index. $\mathrm{php} / \mathrm{revan} / \mathrm{article} / \mathrm{view} / 45 / 09$. Blancas E. Proceso de enseñanza-aprendizaje y nivel de satisfacción de los estudiantes de la Escuela Profesional de Educación Primaria. Horiz la Cienc. 2016; 6(10):205-217.

10. Tolentino L. Desempeño didáctico y académico del docente relacionado a la satisfacción de los estudiantes del programa de complementación pedagógica de la Universidad Nacional Mayor de San Marcos. Tesis para Grado Académico de Magíster en Educación.
Lima, Perú: Universidad Nacional Mayor de San Marcos; 2014. (Fecha de acceso 15 de noviembre del 2017) Disponible en: http://cybertesis.unmsm. edu.pe/bitstream/handle/cybertesis/3981/Tolentino sl.pdf? sequence $=1 \&$ is Allowed $=y$

11. Reymer A. Desempeño docente y satisfacción de los estudiantes del programa de Doctorado en educación de la Universidad Nacional Mayor de san Marcos. Tesis de Doctorado. Lima, Perú: Universidad Nacional Mayor de San Marcos; 2011.

12. Gento S, Vivas M. El SEUE: Un instrumento para conocer la satisfacción de los estudiantes universitarios con su educación. Acción Pedagógica. 2003; 12(2): 16-27.

13. Arregui IG, Chaparro A, López C. Instrumento para evaluar el desempeño docente en educación secundaria desde la percepción de los estudiantes. Colmee. México: $2^{\circ}$ Congreso Latinoamericano de Medición y Evaluación Educativa; 2015.

14. Rivas L. Relación entre el desempeño docente y el logro de objetivos educacionales de estudiantes de la E.A.P. de Enfermería de la UNMSM, 2010. Tesis para Grado. Lima, Perú: Universidad Nacional Mayor de San Marcos; 2010.

15. Montenegro I. Evaluación del desempeño docente. Fundamentos, modelos e instrumentos. Bogotá, Colombia: Corporativa Editorial Magisterio; 2007.

16. Tejedor F, García-Valcárcel A. Evaluación del desempeño docente. 2010;68(247): 439-459. 